\title{
ON GRÜSS TYPE DISCRETE INEQUALITIES
}

\author{
B. G. PACHPATTE
}

Abstract. In this paper we establish two new discrete inequalities of the Grüss type by using a fairly elementary analysis.

Mathematics subject classification (2000): 26D10, 26D15.

Key words and phrases: Grüss type, discrete inequalities, discrete indentities, estimates, modulus, sums.

\section{REFERENCES}

[1] G. GRÜss, Über das maximum des absoluten Betrages von $\frac{1}{b-a} \int_{a}^{b} f(x) g(x) d x-\frac{1}{(b-a)^{2}} \int_{a}^{b} f(x) d x$ $\int_{a}^{b} g(x) d x$, Math. Z. 39 (1935), 215-226.

[2] D. S. Mitrinović, J. E. PeČARIĆ AND A. M. FinK, Classical and new Inequalities in Analysis, Kluwer Academic Publishers, Dordrecht, 1993.

[3] B. G. PACHPATtE, On an inequality of Ostrowski type in three independent variables, J. Math. Anal. Appl. 249 (2000), 583-591.

[4] B. G. PACHPATTE, On a new Ostrowski type inequality in two independent variables, Tomkang J. Math. 32 (2001), 45-49. 